\title{
Surface Probing by Fragment-Based Screening and Computational Methods Identifies Ligandable Pockets on the von Hippel-Lindau (VHL) E3 Ubiquitin Ligase
}

\author{
Xavier Lucas, ${ }^{\dagger}$ İ Inge Van Molle, ${ }^{\ddagger, \S}$ and Alessio Ciulli*, ${ }^{\dagger}$ (i) \\ ${ }^{\dagger}$ Division of Biological Chemistry and Drug Discovery, James Black Centre, School of Life Sciences, University of Dundee, Dow \\ Street, Dundee, DD1 5EH, United Kingdom \\ ${ }^{\ddagger}$ Department of Chemistry, University of Cambridge, Cambridge CB2 1EW, United Kingdom
}

Supporting Information

ABSTRACT: Beyond the targeting of E3 ubiquitin ligases to inhibit protein homeostasis, E3 ligase binders can be repurposed as targeted protein degraders (PROTACs or molecular glues). We sought to identify new binders of the VHL E3 ligase by biophysical fragment-based screening followed by X-ray crystallographic soaking. We identified fragments binding at the ElonginC:Cullin2 interface and a new cryptic pocket in VHL, along with other potential ligandable sites predicted computationally and found to bind solvent molecules in crystal structures. The elucidated interactions provide starting points for future ligand development.

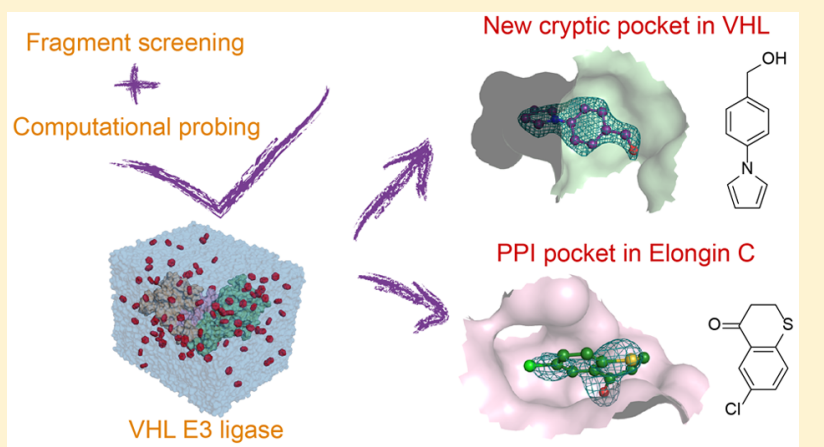

\section{INTRODUCTION}

E3 ubiquitin ligases covalently modify protein substrates by catalyzing ubiquitin transfer from an E2-conjugating enzyme to substrate proteins, thereby marking substrates for proteasomal degradation. ${ }^{1}$ The largest subfamily of E3 ubiquitin ligases are Cullin (Cul)-RING ligases (CRLs), which consist of large modular assemblies comprising a RING domain, a scaffolding $\mathrm{Cul}$ subunit, one or more adaptor proteins, and a substraterecognition subunit that binds to specific protein motifs termed degrons. ${ }^{2,3}$ An archetypical example is the von HippelLindau protein (VHL), which functions as the substratebinding module of CRL2 $2^{\mathrm{VHL}}$, a CRL consisting of VHL, the adaptor proteins Elongin B (EloB) and Elongin C (EloC), $\mathrm{Cul2}$, and Rbx1. VHL features a hydroxyproline (Hyp) recognition site that targets for degradation post-translationally hydroxylated hypoxia-inducible factor (HIF)-1 $\alpha$ subunits. ${ }^{4,5}$ Crystal structures of VHL:EloC:EloB (VCB) in complex with Hyp-containing HIF- $1 \alpha$ peptides, ${ }^{6,7}$ provided the structural basis of Hyp recognition by $\mathrm{VCB}^{8}$ and inspired the design of potent, cell-active Hyp-containing small-molecule inhibitors of VHL. $^{9-12}$

Beyond VHL, the substrate-recognition subunit of only few other CRLs has been targeted using small molecules. ${ }^{3}$ Notably, CRL binders can be converted into targeted protein degraders by conjugating them to a ligand of a protein of interest. ${ }^{13}$ These bivalent compounds, also termed proteolysis-targeting chimeras (PROTACs), have been developed to induce cellular degradation of a wide range of protein targets, including transcription factors, epigenetic targets, and kinases. ${ }^{14-17} \mathrm{We}$ have recently presented the first crystal structure of VCB in complex with a PROTAC and the target protein, highlighting the importance of inducing stable and cooperative proteinprotein recognition around the target E3 ligase site. ${ }^{18}$ Exploiting such ternary complex recognition, we have also shown that $\mathrm{E} 3$ ligase binders can be turned into avid dimerizers that can induce an E3 ligase to destroy itself. ${ }^{19}$ Unlike conventional protein inhibitors, the activity of PROTACs is not dependent upon the biological function or perceived druggability of the attachment sites. ${ }^{13,20}$ Therefore, ligandable pockets in E3 ubiquitin ligases aside from the conventional degron-recognition pocket could potentially be exploited to anchor PROTACs or as molecular glues. ${ }^{3}$ So far, however, ligandable pockets in VCB apart from the HIF-recognition site remain unknown.

The successful small-molecule modulation of the function of specific protein classes historically deemed intractable has motivated a growing interest in developing technologies to probe protein surfaces and identify secondary binding sites. $^{21,22}$ In particular, fragment screening by X-ray crystallography and NMR has seen widespread application. ${ }^{23-26}$ From a computational perspective, a variety of empirical-, grid-, and force-field based methods have been developed. ${ }^{27}$ Tools such as SiteMap ${ }^{28}$ and FTMap ${ }^{29,30}$ are routinely used to predict and analyze protein-ligand and protein-protein interfaces ${ }^{31,32}$ as well as to study cryptic pockets. ${ }^{33}$ Mixed-solvent molecular dynamics (MD) simulations enable solvent mapping with protein flexibility and explicit water, albeit at a substantial

Received: May 28, 2018

Published: July 24, 2018 
A
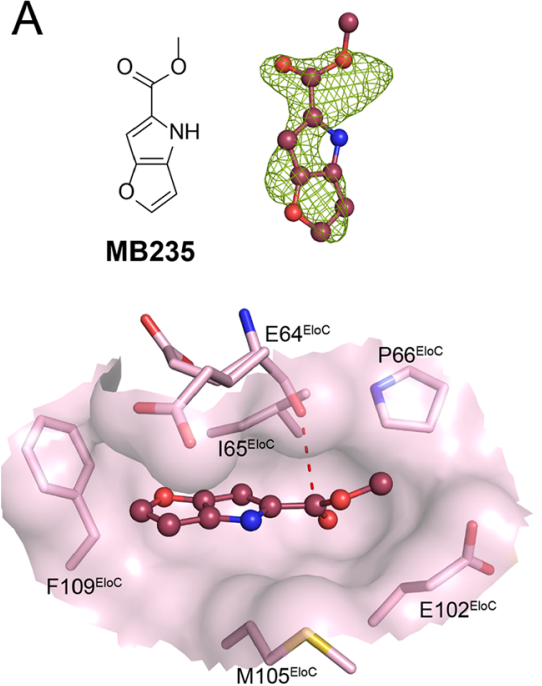

B

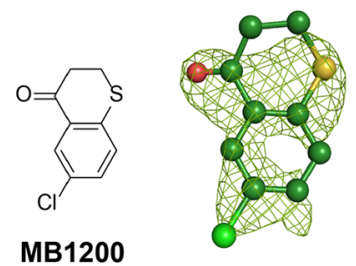

MB1200

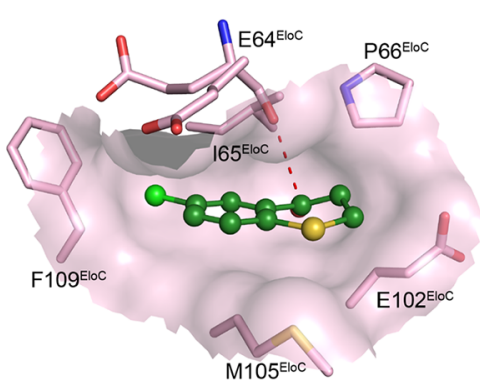

C

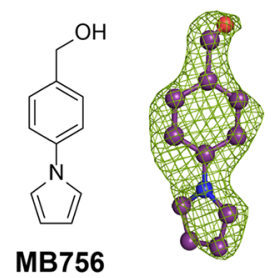

D

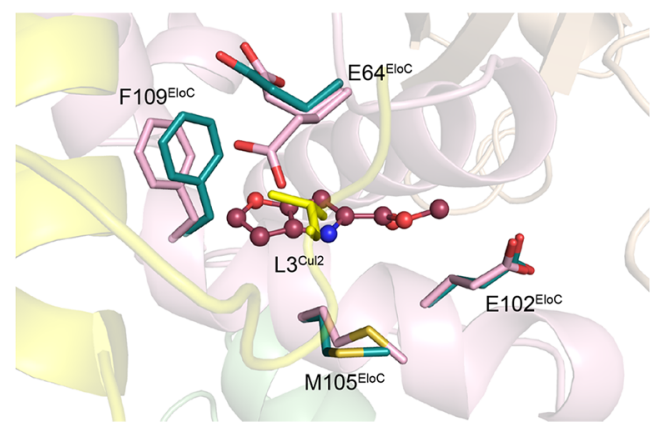

E
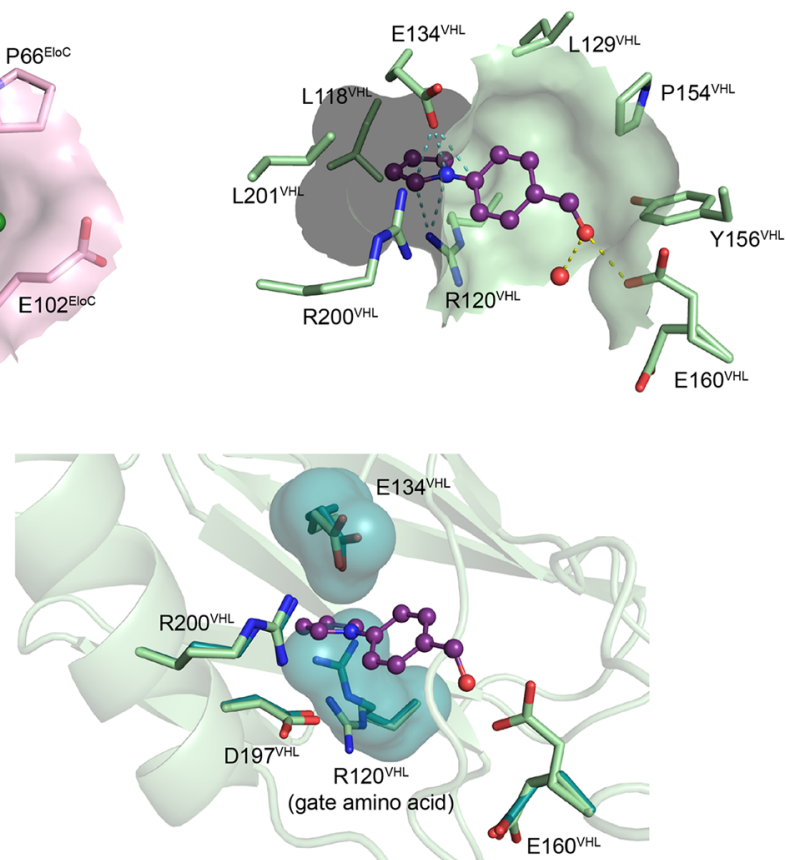

Figure 1. Fragment-based probing of the VHL:EloC:EloB E3 ubiquitin ligase. Chemical structure, $F_{0}-F_{c}$ electron density omit map contoured at $3 \sigma$ level, and X-ray crystal structure of fragment (A) MB235 in complex with VCB (PDB 6GMN), (B) MB1200 in complex with VCB (PDB $6 \mathrm{GMX}$ ), and (C) MB756 in complex with $\mathrm{VCBH}$ (PDB 6GMR). Hydrogen bonds, ion $-\pi$ interactions, and $\mathrm{C}=\mathrm{O} \cdots \mathrm{C}=\mathrm{O}$ contacts are represented as yellow, cyan, and red dashed lines, respectively. (D) Superposition of the crystal structure of VCB in complex with MB235 and with Cul2 (PDB 4WQO), ${ }^{39}$ colored deep teal and yellow. L3 ${ }^{\text {Cul2 }}$ and fragments MB235 and MB1200 occupy the same pocket in EloC. (E) Superposition of the crystal structure of VCBH in complex with MB756 and of VCBH alone (PDB 4AJY), ${ }^{11}$ colored deep teal. Note that formation of the cryptic pocket requires rearrangement of the gate amino acid $\mathrm{R} 120^{\mathrm{VHL}}$.

computational cost. ${ }^{34}$ This method has also been used to detect and study allosteric sites, cryptic pockets, and hotspots of intermolecular interaction. ${ }^{34-38}$

Herein we probe the surface of VCB to identify binding sites by fragment-based screening and computational pocket detection methods. The screening hits were validated by $\mathrm{X}$ ray crystallography, which revealed two novel ligandable pockets: one involving the EloC:Cul interface and the other involving a previously unknown cryptic pocket in VHL. In addition, computational approaches located other potential sites in VCB that bind solvent molecules in available crystallographic data, suggesting that they could also be ligandable.

\section{RESULTS AND DISCUSSION}

The biophysical cascade consisted of a primary screen of over 1200 rule of three-compliant fragments from the Maybridge library using differential scanning fluorimetry (DSF) and of 144 fragments randomly picked from the same library using one-dimensional ${ }^{1} \mathrm{H}$ NMR spectroscopy. ${ }^{40}$ Screening hits were validated by NMR spectroscopy and subsequent X-ray crystallography (Supporting Information (SI), Figure S1).

DSF monitors the unfolding temperature of a protein using a fluorescent dye that preferentially binds to unfolded proteins.
We screened our fragment library, assayed as singletons, against both VCB and VCB preincubated with HIF-1 $\alpha$ peptide $(\mathrm{VCBH})$. We reasoned that this approach could aid identification and direct discrimination between binders of the HIF site and binders elsewhere in the complex. DSF hits were defined as fragments resulting in an increase in melting temperature $\left(\Delta T_{\mathrm{m}}=T_{\mathrm{m} \text {,protein+fragment }}-T_{\mathrm{m} \text {,protein }}\right)$ of either $\mathrm{VCB}$ or $\mathrm{VCBH}$ greater than $0.5^{\circ} \mathrm{C}$. In addition, five fragments were considered hits due to their effect on the overall melting properties of VCB. In total, 65 unique hits were identified by DSF (5.2\% overall hit rate). In parallel, the ligand-based NMR screen was performed in cocktails of three fragments. We carried out a series of one-dimensional ${ }^{1} \mathrm{H}$ NMR spectroscopy binding experiments: water ligand observed gradient spectroscopy (waterLOGSY), saturation transfer difference (STD), and Carr-Purcell-Meiboom-Gill (CPMG) relaxation-edited sequences. ${ }^{41}$ In these experiments, binding was assessed by comparing the proton signals of the fragments in the presence and in the absence of VCB. Specific binding to the VHL:HIF interface was probed by subsequently monitoring displacement of the ligand signals using the HIF- $1 \alpha$ peptides 10-mer DEALA-Hyp-YIPD or 19-mer DEALA-Hyp-YIPMDDDFQLRSF ( $K_{\mathrm{d}}$ of 180 or $3 \mathrm{nM}$, respectively)., ${ }^{911}$ Fragments were considered hits in the NMR screen if they showed 


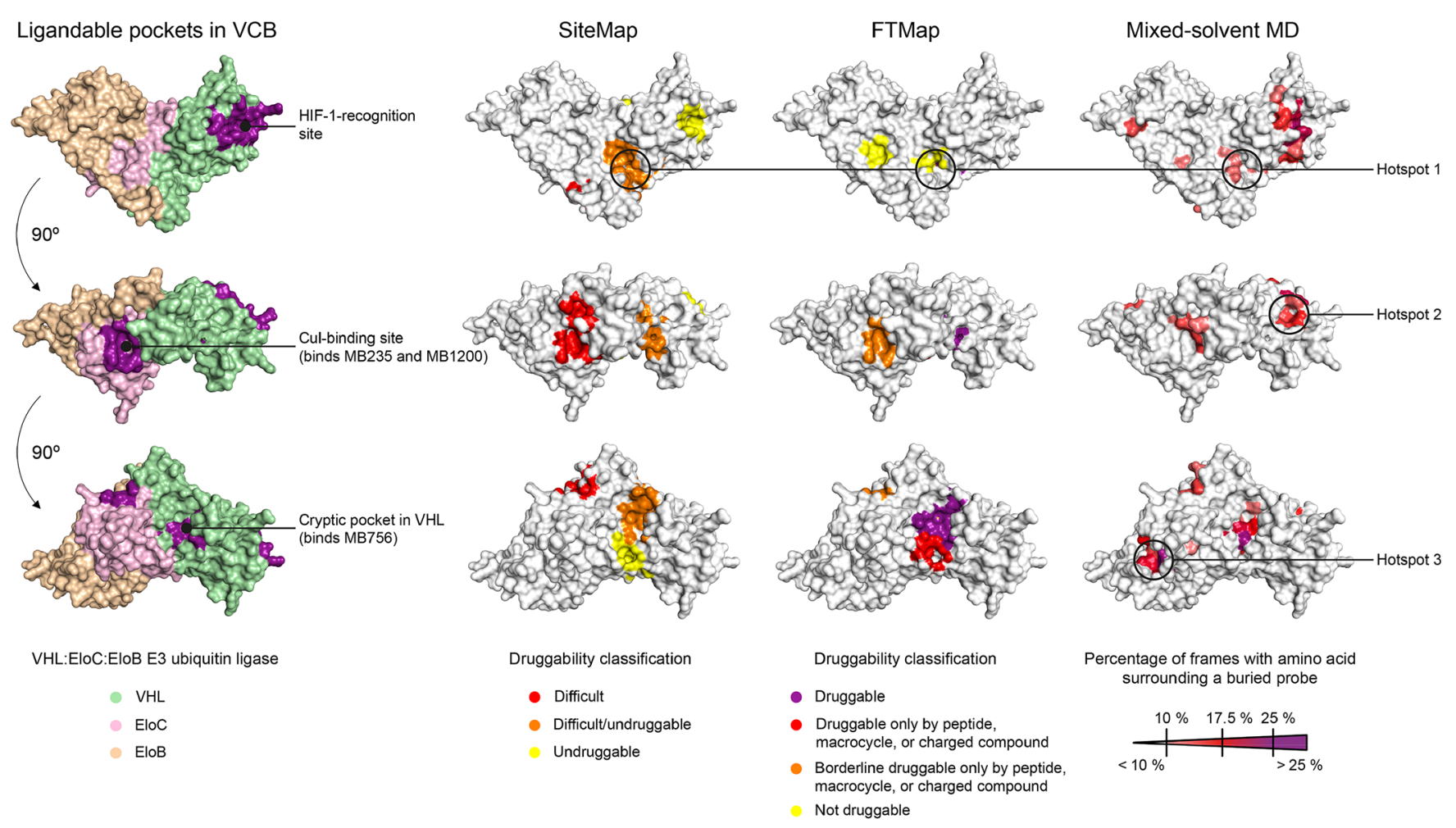

Figure 2. Computational surface probing of the VHL:EloC:EloB E3 ligase. Ligandable pockets in VCB and sites predicted by SiteMap, ${ }^{28}$ FTMap, ${ }^{29,30}$ and mixed-solvent MD are highlighted. Pockets identified by SiteMap and FTMap are colored according to druggability classification. Consensus amino acids identified by mixed-solvent MD are colored according to the percentage of frames in the mixed-solvent MD simulation they are in contact with a buried probe. Predicted hotspots 1-3 are highlighted and labeled.

binding in at least two of the three NMR experiments. This resulted in 17 unique hits ( $11.8 \%$ overall hit rate).

In total, 82 fragments were selected as hits from these two primary screens and subsequently validated by a second round of NMR experiments (waterLOGSY, STD, and CPMG) as cocktails of two for the DSF hits and as singletons for the NMR hits. This secondary screen validated 18 of the 82 hits identified in the primary screen $(78.0 \%$ attrition rate). Notably, all but one of the validated fragment hits were not displaced by the HIF- $1 \alpha$ peptide, suggesting that they may bind to other sites on the VCB complex. To locate those potential new sites, we soaked the 18 validated fragment hits in both VCB and VCBH crystals, which have distinct crystal packing $\left(P 4_{1} 22\right.$ for $\mathrm{VCB}$ and $\mathrm{P}_{3} 2_{1} 2$ for $\mathrm{VCBH}$ ). This maximizes the likelihood of success in the crystallographic fragment soaking by increasing the combined solvent-exposed surface of the complex. The soaking experiment yielded three fragments binding to sites in VCB other than the HIF-recognition site in VHL (Figure 1 and SI, Figure S2 and Table S1), consistent with the NMR data showing lack of competition by the HIF- $1 \alpha$ peptide or a Hyp-containing fragment binding weakly at the HIF site. ${ }^{11}$ Fragments MB235 and MB1200 (PDB 6GMN and 6GMX, $\Delta T_{\mathrm{m}}=0.7$ and $1.2{ }^{\circ} \mathrm{C}$ against $\mathrm{VCBH}$, respectively) bind to a hydrophobic cleft in EloC that is only accessible in the VCB crystals and occluded by crystal packing contacts in VCBH. The newly identified binding site is defined by EloC residues E64, I65, P66, E102, M105, A106, and F109. Both compounds share an aromatic portion that forms hydrophobic contacts and a carbonyl group that occupies the same position in the two crystal structures. The carbonyl carbon of the fragments engages in a $\mathrm{C}=\mathrm{O} \cdots \mathrm{C}=\mathrm{O}$ contact with the backbone carbonyl oxygen of E64 ${ }^{\mathrm{EloC}}$. Additionally, we observe flexibility of the $\mathrm{E} 64^{\mathrm{EloC}}$ side chain in the cocrystal structures (Figure 1A,B). Superposition of EloC in complex with the fragments and with $\mathrm{Cul} 2$ reveals that the fragments bind to the same cavity used for recognition of $\mathrm{L}_{3}{ }^{\mathrm{Cul} 2}$ in the EloC:Cul2 interface (Figure 1D), which we have recently probed using low-affinity peptides. $^{42}$ In contrast, MB756 binds to both VCB and VCBH crystals (PDB 6GMQ and 6GMR, respectively, $\Delta T_{\mathrm{m}}=1.3{ }^{\circ} \mathrm{C}$ against VCB) in a previously unknown cryptic pocket in VHL, located over $15 \AA$ away from the HIF-recognition site (Figure 2). The pyrrole ring of MB756 is inserted in a pocket in VHL formed by VHL residues L118, F119, R120, G127, L128, L129, E134, D197, and L201. The pocket is accessible only after rearrangement of $\mathrm{R} 120^{\mathrm{VHL}}$, which acts as a gate amino acid to accommodate the fragment (Figure 1E). Upon binding, the pyrrole ring of the ligand engages in ion $-\pi$ interactions with $\mathrm{R} 120^{\mathrm{VHL}}$ and $\mathrm{E} 134^{\mathrm{VHL}}$, and the phenolic hydroxyl group protrudes away from the pocket to form hydrogen bonds to the side chain of $\mathrm{E} 160^{\mathrm{VHL}}$ and to a water molecule (Figure 1C). We used isothermal titration calorimetry (ITC) to characterize the binding affinity of the fragments to VCB (SI, Figure S3). Dissociation constants of 5.0 and $6.7 \mathrm{mM}$ were obtained for MB756 and MB1200, respectively, resulting in a ligand efficiency (LE) of 0.24 and $0.25 \mathrm{kcal} / \mathrm{mol} \cdot \mathrm{heavy}$ atom, respectively. We have previously shown that fragments in this range of affinity and LE can be elaborated into high affinity binders. ${ }^{43}$ Together, the results support specific binding interactions and qualify these pockets as ligandable, at least to weak-affinity fragments.

The newly discovered pockets in VCB are of substantial interest in several research areas. First, we prove that the $\mathrm{Cul}$ interface of EloC is ligandable and provide the first ligandbound structures of EloC. Second, the cryptic pocket in VHL 
A

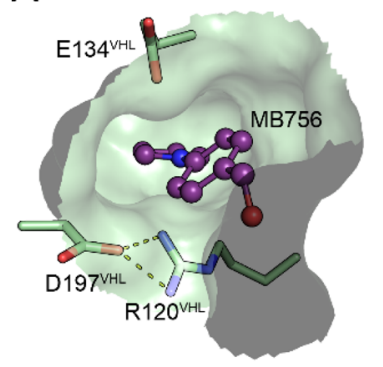

B

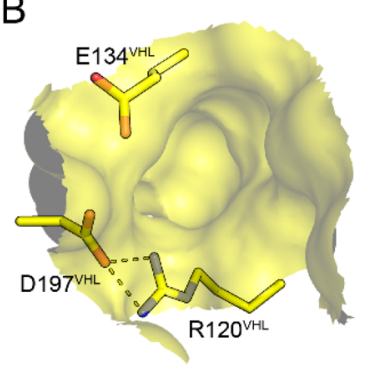

C

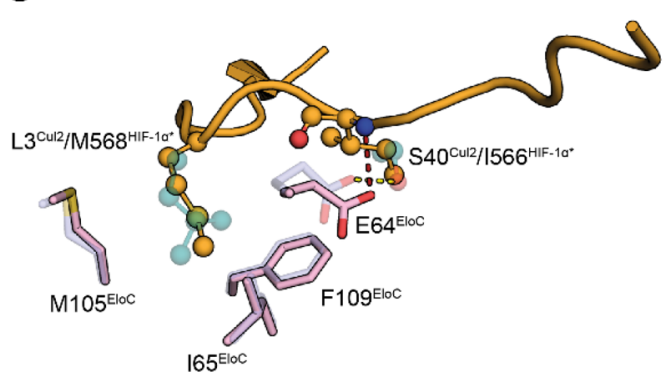

D

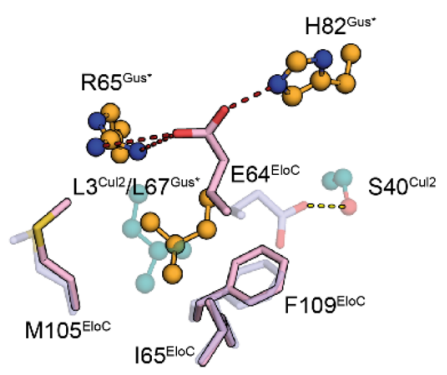

E

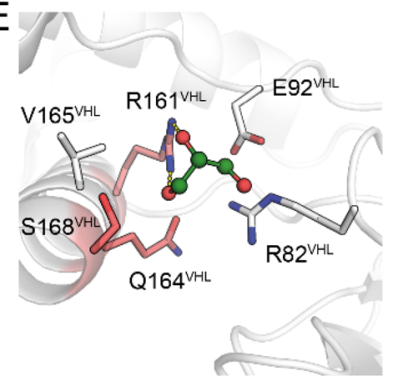

F

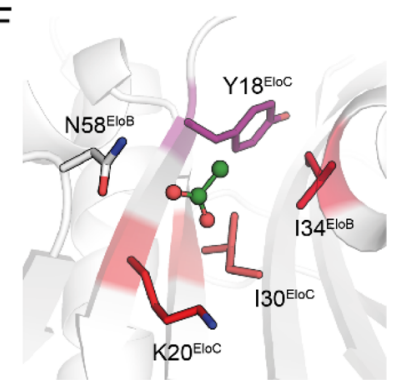

Figure 3. Structural analysis of VHL:EloC:EloB E3 ubiquitin ligase sites. Surface representation of the cryptic pocket in VHL in (A) the VCBH:MB756 cocrystal structure (PDB 6GMR) and in (B) a frame extracted from the MD trajectory of VCB cosolvated with dioxopiperazine. Cul site in EloC upon superposition of VCB:Cul2 (PDB 4WQO), ${ }^{39}$ shown fogged, with (C) VCBH (PDB 4AJY) ${ }^{11}$ and with (D) Gustavus:EloCB (PDB 2FNJ). ${ }^{47}$ Crystal structure of VCB with (E) glycerol in hotspot 1 (PDB 4AJY) and (F) acetate in hotspot 3 (PDB 4B9K). ${ }^{10}$ In (B), note the rearrangement of gate amino acid $\mathrm{R} 120^{\mathrm{VHL}}$. In (C) and (D), amino acids of the symmetry-related protomer in the crystal are labeled with $*$ and colored bright orange. (E,F) Amino acids are colored according to the percentage of frames in the mixed-solvent MD simulation they are in contact with a buried probe, as in Figure 2. Hydrogen bonds and salt bridges are shown as yellow dashed lines, and in (C) and (D) they are colored red if they occur as part of a crystal contact.

involves the most frequently occurring mutation in Chuvash polycythemia, R200W, which prevents degradation of phosphorylated tyrosine-protein kinase JAK2 through the proteasome. $^{44,45}$ Additionally, MB756 also contacts the conserved $\mathrm{P} 154^{\mathrm{VHL}}$, which has been proposed to play an important role in substrate poly ubiquitylation by CRLs. ${ }^{46}$ Thus, elaborated higher-affinity binders could serve as chemical biology tools to study E3 ligase activity and the Chuvash disease at the molecular level. Notably, optimized compounds binding to the new sites could also be converted into targeted protein degraders, i.e., PROTACs. ${ }^{13}$ The presence of several ligandable pockets in VCB additionally sets an ideal, yet challenging, scenario to explore these and other binding sites using computational pocket detection methods. Thus, we next subjected VCB to a surface probing campaign using SiteMap, ${ }^{28}$ FTMap, ${ }^{29,30}$ and mixed-solvent $\mathrm{MD}^{34}$

In the case of SiteMap and FTMap, we processed the whole VCB complex using standard parameters. For the mixedsolvent $\mathrm{MD}$, we first selected 15 fragment-size molecules with varied physicochemical properties, chemotypes, and molecular sizes as probes. We reasoned that exploration of protein surfaces beyond druggability considerations requires thoughtful yet bold probe selection. With this in mind, apart from traditional probes typically used in solvent mapping, such as benzene and isobutanol, we also considered biomimetics of peptidic bonds, a zwitterionic species, a capped alanine, a biaryl, a fluorinated probe, and a sulfonamide (SI, Figure S4 and Table S2). Each probe was used to cosolvate VCB, and the whole system was then subjected to MD simulations. Potential binding sites were identified by recording consensus amino acids surrounding probes buried in the protein surface during the simulations.
Ligandable sites in VCB and those predicted computationally are highlighted in Figure 2. The three pockets validated experimentally provided a suitable opportunity to benchmark the computational results. SiteMap and FTMap were very successful in locating precisely the binding site of MB235 and MB1200, and the MD approach suggested the importance of surrounding amino acids $\mathrm{M}_{105^{\mathrm{EloC}}}$ and $\mathrm{D} 179^{\mathrm{VHL}}$ in noncovalent recognition (SI, Figures S8 and S9). Consistent with these predictions, an M105 $\mathrm{A}^{\text {EloC }}$ mutant resulted in a 35 -fold decrease in binding affinity toward Cul2 compared to wild-type EloC, ${ }^{48}$ and a $\mathrm{K} 4 \mathrm{~A}^{\mathrm{Cul2}}$ mutant peptide, which would prevent a salt bridge between $\mathrm{K} 4^{\mathrm{Cul} 2}$ and $\mathrm{D} 179^{\mathrm{VHL}}$, exhibited no binding to $\mathrm{VCB}^{42}$ The HIF-recognition site in VHL was properly detected by the mixed-solvent MD method. However, FTMap failed to locate it and SiteMap perceived it as undruggable, consistent with the observation that only one fragment targeting the HIF site emerged from our biophysical screen (SI, Figure S1) and with the challenges in detecting binding of fragments resulting from deconstructing ligands targeting this interface. ${ }^{43}$ The methods also predicted a potentially druggable large spot surrounding the cryptic pocket in VHL, suggesting that there is promise in developing MB756 into a smallmolecule binder by engaging in farther favorable contacts (SI, Figure $\mathrm{S} 10$ ). Because mixed-solvent $\mathrm{MD}$ can reveal cryptic pockets, $^{36,38}$ we next investigated whether the simulations captured formation of the MB756 cavity in VHL. Indeed, cosolvation of $\mathrm{VCB}$ with dioxopiperazine induced opening of the pocket by triggering the required rearrangement of $\mathrm{R} 120^{\mathrm{VHL}}$ (Figure 3A,B). However, the probes did not occupy the cavity during the simulations, presumably because of its hydrophobic nature (Figure 1C).

Apart from detecting the three ligandable sites in VCB, we were also interested in locating pockets elsewhere in the 
protein surface. SiteMap and FTMap proposed an additional, albeit low-druggable, site in VHL (labeled as "hotspot 1 " in Figure 2). The same pocket was also identified by mixedsolvent $\mathrm{MD}$, as well as two unique sites in VHL and EloBC ("hotspot 2" and "hotspot 3", respectively). We mined the Protein Data Bank (PDB) for crystal structures of VHL, EloC, and EloB, aiming at identifying intermolecular contacts and nonmodeled electron density in hotspots 1-3. Additionally, we also inspected the binding sites of MB235, MB756, and MB1200 for contacts that could inform future structure-based fragment growing endeavors.

We gathered a total of 45 crystal structures: 36 crystal structures of VCB and 9 crystal structures of complexes of EloBC with viral factors or substrate-recognition subunits other than VHL (SI, Table S5). While we could not identify contacts involving the MB756 cryptic pocket, crystal contacts were found in the HIF-recognition site (SI, Figure S11) and the MB235/MB1200 pocket in EloC. In VCB:HIF-1 $\alpha$ peptide crystal structures, the EloC pocket is occupied by M568 ${ }^{\mathrm{HIF}-1 \alpha}$ of a symmetry-related protomer (Figure 3C). In addition, the side chain of $\mathrm{E} 64^{\mathrm{EloC}}$, which assists in Cul2-recognition by VCB by hydrogen-bonding to $S 40^{\mathrm{Cul} 2}$, engages in a hydrogen bond with the backbone of $\mathrm{I} 566^{\mathrm{HIF}-1 \alpha}$. Similarly, in a crystal structure of EloBC in complex with Gustavus (Gus), which is the substrate-recognition subunit of the CRL5 ${ }^{\text {Gus }}$ E3 ubiquitin ligase, the same cavity is occupied by $\mathrm{L} 67^{\mathrm{Gus}}$ of a symmetryrelated unit, whereas E64 ${ }^{\text {EloC }}$ flips and interacts with R65 ${ }^{\text {Gus }}$ and $\mathrm{H} 82^{\text {Gus }}$ (Figure 3D). Notably, side chain flexibility of E64 ${ }^{\mathrm{EloC}}$ is also observed in the presence of fragments MB235 and MB1200 (Figure 1A,B).

We extended the analysis to hotspots 1-3 predicted computationally (Figure 2). We found a crystal structure of VCB with glycerol bound to hotspot 1 , sandwiched in a polar cleft and engaging in two hydrogen bonds with R161 ${ }^{\mathrm{VHL}}$ (Figure 3E). In hotspot 2, we found crystal structures of VCB with an elongated blob of nonmodeled electron density that did not match a water molecule, suggesting that a small solvent molecule may be trapped in the cavity (SI, Figure S12). We also found several crystals of VCB in complex with HIF small-molecule mimetics with a water molecule located in hotspot 3. In some cases, the water is displaced by an acetate that satisfies hydrophobic contacts with $\mathrm{Y} 18^{\mathrm{EloC}}, \mathrm{I} 30^{\mathrm{EloC}}$, and I34 ${ }^{\text {EloB }}$ (Figure 3F).

\section{CONCLUSIONS}

In summary, we report crystal structures of the VHL:EloC:EloB E3 ubiquitin ligase in complex with fragment-based screening hits. Two fragments bound to a small cavity at the EloC:Cul interface, whereas another fragment bound to a novel cryptic pocket in VHL. We also subject the VCB complex to computational surface probing and study all binding sites by systematic analysis of available crystal structures of VHL, EloC, and EloB. We identify crystal contacts in the EloC pocket that could inform future fragment elaboration as well as solvents bound to potential hotspots proposed computationally. Additionally, we detect formation of the VHL cryptic pocket during the MD simulations by rearrangement of the gate amino acid $\mathrm{R} 120^{\mathrm{VHL}}$, as observed crystallographically.

The discovered cryptic pocket in VHL involves R200 $\mathrm{VHL}$, which is highly mutated in Chuvash polycythemia, and $\mathrm{P} 154^{\mathrm{VHL}}$, which has been proposed to play an important role in substrate poly ubiquitylation by CRLs. ${ }^{45,46}$ The presented fragment-bound structures of VCB will guide the development and optimization of more potent ligands. ${ }^{43}$ Binders of the cryptic pocket could be optimized into an allosteric ligand or stabilizing probe for the Chuvash mutant protein, whereas EloC binders could be used to disrupt the assembly of CRL2 ligases $^{49}$ and to study the biology of EloC-containing E3 ligases. Additionally, elaborated binders of the newly identified ligandable sites could also be converted into targeted protein degraders. Indeed, the realization that persistent and cooperative de novo protein-protein recognition, rather than target binding affinity, dictates preferential substrate degradation, ${ }^{18}$ and that suboptimal VHL ligands can render very potent degraders, ${ }^{50}$ suggests that even weak binders of secondary pockets in E3 ligases could be used as anchoring ligands for PROTAC conjugation.

\section{EXPERIMENTAL SECTION}

Tested compounds were purchased and have a purity $\geq 95 \%$ (HPLC analysis).

\section{ASSOCIATED CONTENT}

\section{Supporting Information}

The Supporting Information is available free of charge on the ACS Publications website at DOI: 10.1021/acs.jmedchem.8b00842.

Experimental section: protein expression and purification, DSF, ligand-observed NMR spectroscopy, crystallization and structure determination, ITC, computational methods; figures and tables (PDF) Molecular formula strings CSV)

\section{Accession Codes}

Atomic coordinates have been deposited in the Protein Data Bank and will be released upon article publication. Accession codes of VCB in complex with MB235 (PDB 6GMN), MB756 (PDB 6GMQ), MB1200 (PDB 6GMX), and $\mathrm{VCBH}$ in complex with MB756 (PDB 6GMR).

\section{AUTHOR INFORMATION}

\section{Corresponding Author}

*Phone: +441382386230. Fax: +441382386373. E-mail: a. ciulli@dundee.ac.uk.

\section{ORCID}

Xavier Lucas: 0000-0003-0887-3976

Alessio Ciulli: 0000-0002-8654-1670

\section{Present Address}

${ }^{\S}$ I.V.M.: VIB-VUB Center for Structural Biology, Structural Biology Brussels, Vrije Universiteit Brussel, Pleinlaan 2, 1050 Brussels, Belgium

\section{Author Contributions}

The manuscript was written through contributions of all authors. All authors have given approval to the final version of the manuscript. X.L. and I.V.M. contributed equally.

Notes

The authors declare no competing financial interest.

\section{ACKNOWLEDGMENTS}

Funding support is gratefully acknowledged from the European Research Council (ERC-2012-StG-311460 DrugE3CRLs Starting Grant to A.C.), the European Commission (H2020MSCA-IF-2015-806323 Marie Skłodowska-Curie Actions Individual Fellowship to X.L. and EC PIEF-GA-2010-275683 
Marie-Curie Intra European Fellowship to I.V.M.), and the Wellcome Trust (Strategic Awards 100476/Z/12/Z for biophysics and drug discovery and 094090/Z/10/Z for structural biology and X-ray crystallography to the Division of Biological Chemistry and Drug Discovery at Dundee). We thank Professor Chris Abell (Department of Chemistry, Cambridge) for access to the fragment library, and the Soleil and Diamond Light Source for beamtime and beamline support at beamlines Proxima 1, i03, and i24.

\section{ABBREVIATIONS USED}

CRL, Cullin-RING E3 ubiquitin ligase; Cul, Cullin; DSF, differential scanning fluorimetry; CPMG, Carr-PurcellMeiboom-Gill; EloB, elongin B; EloC, elongin C; Gus, Gustavus protein; HIF, hypoxia-inducible factor; Hyp, hydroxyproline; ITC, isothermal titration calorimetry; LE, ligand efficiency; MD, molecular dynamics; PROTAC, proteolysis-targeting chimera; PDB, Protein Data Bank; STD, saturation transfer difference; $T_{\mathrm{m}}$, melting temperature; VHL, von Hippel-Lindau protein; VCB, VHL:EloC:EloB; VCBH, VCB:HIF-1 $\alpha$ complex; waterLOGSY, water ligand observed gradient spectroscopy

\section{REFERENCES}

(1) Hershko, A.; Ciechanover, A. The ubiquitin system. Annu. Rev. Biochem. 1998, 67, 425-479.

(2) Lydeard, J. R.; Schulman, B. A.; Harper, J. W. Building and remodelling Cullin-RING E3 ubiquitin ligases. ЕMBO Rep. 2013, 14, 1050-1061.

(3) Lucas, X.; Ciulli, A. Recognition of substrate degrons by E3 ubiquitin ligases and modulation by small-molecule mimicry strategies. Curr. Opin. Struct. Biol. 2017, 44, 101-110.

(4) Ivan, M.; Kondo, K.; Yang, H.; Kim, W.; Valiando, J.; Ohh, M.; Salic, A.; Asara, J. M.; Lane, W. S.; Kaelin, W. G., Jr. HIF $\alpha$ targeted for VHL-mediated destruction by proline hydroxylation: implications for $\mathrm{O}_{2}$ sensing. Science 2001, 292, 464-468.

(5) Jaakkola, P.; Mole, D. R.; Tian, Y. M.; Wilson, M. I.; Gielbert, J.; Gaskell, S. J.; von Kriegsheim, A.; Hebestreit, H. F.; Mukherji, M.; Schofield, C. J.; Maxwell, P. H.; Pugh, C. W.; Ratcliffe, P. J. Targeting of HIF- $\alpha$ to the von Hippel-Lindau ubiquitylation complex by $\mathrm{O}_{2}-$ regulated prolyl hydroxylation. Science 2001, 292, 468-472.

(6) Min, J. H.; Yang, H.; Ivan, M.; Gertler, F.; Kaelin, W. G., Jr.; Pavletich, N. P. Structure of an HIF- $1 \alpha$-pVHL complex: hydroxyproline recognition in signaling. Science 2002, 296, 1886-1889.

(7) Hon, W. C.; Wilson, M. I.; Harlos, K.; Claridge, T. D.; Schofield, C. J.; Pugh, C. W.; Maxwell, P. H.; Ratcliffe, P. J.; Stuart, D. I.; Jones, E. Y. Structural basis for the recognition of hydroxyproline in HIF-1 $\alpha$ by pVHL. Nature 2002, 417, 975-978.

(8) Loenarz, C.; Mecinovic, J.; Chowdhury, R.; McNeill, L. A.; Flashman, E.; Schofield, C. J. Evidence for a stereoelectronic effect in human oxygen sensing. Angew. Chem., Int. Ed. 2009, 48, 1784-1787.

(9) Buckley, D. L.; Van Molle, I.; Gareiss, P. C.; Tae, H. S.; Michel, J.; Noblin, D. J.; Jorgensen, W. L.; Ciulli, A.; Crews, C. M. Targeting the von Hippel-Lindau E3 ubiquitin ligase using small molecules to disrupt the VHL/HIF- $1 \alpha$ interaction. J. Am. Chem. Soc. 2012, 134, $4465-4468$.

(10) Buckley, D. L.; Gustafson, J. L.; Van Molle, I.; Roth, A. G.; Tae, H. S.; Gareiss, P. C.; Jorgensen, W. L.; Ciulli, A.; Crews, C. M. Smallmolecule inhibitors of the interaction between the E3 ligase VHL and HIF-1 $\alpha$. Angew. Chem., Int. Ed. 2012, 51, 11463-11467.

(11) Van Molle, I.; Thomann, A.; Buckley, D. L.; So, E. C.; Lang, S.; Crews, C. M.; Ciulli, A. Dissecting fragment-based lead discovery at the von Hippel-Lindau protein:hypoxia inducible factor $1 \alpha$ proteinprotein interface. Chem. Biol. 2012, 19, 1300-1312.

(12) Galdeano, C.; Gadd, M. S.; Soares, P.; Scaffidi, S.; Van Molle, I.; Birced, I.; Hewitt, S.; Dias, D. M.; Ciulli, A. Structure-guided design and optimization of small molecules targeting the proteinprotein interaction between the von Hippel-Lindau (VHL) E3 ubiquitin ligase and the hypoxia inducible factor (HIF) $\alpha$ subunit with in vitro nanomolar affinities. J. Med. Chem. 2014, 57, 8657-8663.

(13) Raina, K.; Crews, C. M. Targeted protein knockdown using small molecule degraders. Curr. Opin. Chem. Biol. 2017, 39, 46-53.

(14) Zengerle, M.; Chan, K. H.; Ciulli, A. Selective small molecule induced degradation of the BET bromodomain protein BRD4. ACS Chem. Biol. 2015, 10, 1770-1777.

(15) Bondeson, D. P.; Mares, A.; Smith, I. E.; Ko, E.; Campos, S.; Miah, A. H.; Mulholland, K. E.; Routly, N.; Buckley, D. L.; Gustafson, J. L.; Zinn, N.; Grandi, P.; Shimamura, S.; Bergamini, G.; FaelthSavitski, M.; Bantscheff, M.; Cox, C.; Gordon, D. A.; Willard, R. R.; Flanagan, J. J.; Casillas, L. N.; Votta, B. J.; den Besten, W.; Famm, K.; Kruidenier, L.; Carter, P. S.; Harling, J. D.; Churcher, I.; Crews, C. M. Catalytic in vivo protein knockdown by small-molecule PROTACs. Nat. Chem. Biol. 2015, 11, 611-617.

(16) Winter, G. E.; Buckley, D. L.; Paulk, J.; Roberts, J. M.; Souza, A.; Dhe-Paganon, S.; Bradner, J. E. Drug development. Phthalimide conjugation as a strategy for in vivo target protein degradation. Science 2015, 348, 1376-1381.

(17) Robb, C. M.; Contreras, J. I.; Kour, S.; Taylor, M. A.; Abid, M.; Sonawane, Y. A.; Zahid, M.; Murry, D. J.; Natarajan, A.; Rana, S. Chemically induced degradation of CDK9 by a proteolysis targeting chimera (PROTAC). Chem. Commun. (Cambridge, U. K.) 2017, 53, $7577-7580$.

(18) Gadd, M. S.; Testa, A.; Lucas, X.; Chan, K. H.; Chen, W.; Lamont, D. J.; Zengerle, M.; Ciulli, A. Structural basis of PROTAC cooperative recognition for selective protein degradation. Nat. Chem. Biol. 2017, 13, 514-521.

(19) Maniaci, C.; Hughes, S. J.; Testa, A.; Chen, W.; Lamont, D. J.; Rocha, S.; Alessi, D. R.; Romeo, R.; Ciulli, A. Homo-PROTACs: bivalent small-molecule dimerizers of the VHL E3 ubiquitin ligase to induce self-degradation. Nat. Commun. 2017, 8, 830.

(20) Shimokawa, K.; Shibata, N.; Sameshima, T.; Miyamoto, N.; Ujikawa, O.; Nara, H.; Ohoka, N.; Hattori, T.; Cho, N.; Naito, M. Targeting the allosteric site of oncoprotein BCR-ABL as an alternative strategy for effective target protein degradation. ACS Med. Chem. Lett. 2017, 8, 1042-1047.

(21) Guarnera, E.; Berezovsky, I. N. Allosteric sites: remote control in regulation of protein activity. Curr. Opin. Struct. Biol. 2016, 37, 18 .

(22) Knapp, S. Emerging target families: intractable targets. Handb. Exp. Pharmacol. 2015, 232, 43-58.

(23) Hajduk, P. J.; Huth, J. R.; Fesik, S. W. Druggability indices for protein targets derived from NMR-based screening data. J. Med. Chem. 2005, 48, 2518-2525.

(24) Edfeldt, F. N.; Folmer, R. H.; Breeze, A. L. Fragment screening to predict druggability (ligandability) and lead discovery success. Drug Discovery Today 2011, 16, 284-287.

(25) Ludlow, R. F.; Verdonk, M. L.; Saini, H. K.; Tickle, I. J.; Jhoti, $\mathrm{H}$. Detection of secondary binding sites in proteins using fragment screening. Proc. Natl. Acad. Sci. U. S. A. 2015, 112, 15910-15915.

(26) Amato, A.; Lucas, X.; Bortoluzzi, A.; Wright, D.; Ciulli, A. Targeting ligandable pockets on plant homeodomain (PHD) zinc finger domains by a fragment-based approach. ACS Chem. Biol. 2018, 13, 915-921.

(27) Wagner, J. R.; Lee, C. T.; Durrant, J. D.; Malmstrom, R. D.; Feher, V. A.; Amaro, R. E. Emerging computational methods for the rational discovery of allosteric drugs. Chem. Rev. 2016, 116, 63706390.

(28) Halgren, T. A. Identifying and characterizing binding sites and assessing druggability. J. Chem. Inf. Model. 2009, 49, 377-389.

(29) Brenke, R.; Kozakov, D.; Chuang, G. Y.; Beglov, D.; Hall, D.; Landon, M. R.; Mattos, C.; Vajda, S. Fragment-based identification of druggable 'hot spots' of proteins using Fourier domain correlation techniques. Bioinformatics 2009, 25, 621-627.

(30) Kozakov, D.; Hall, D. R.; Chuang, G. Y.; Cencic, R.; Brenke, R.; Grove, L. E.; Beglov, D.; Pelletier, J.; Whitty, A.; Vajda, S. Structural 
conservation of druggable hot spots in protein-protein interfaces. Proc. Natl. Acad. Sci. U. S. A. 2011, 108, 13528-13533.

(31) Vidler, L. R.; Brown, N.; Knapp, S.; Hoelder, S. Druggability analysis and structural classification of bromodomain acetyl-lysine binding sites. J. Med. Chem. 2012, 55, 7346-7359.

(32) Kozakov, D.; Hall, D. R.; Jehle, S.; Luo, L.; Ochiana, S. O.; Jones, E. V.; Pollastri, M.; Allen, K. N.; Whitty, A.; Vajda, S. Ligand deconstruction: Why some fragment binding positions are conserved and others are not. Proc. Natl. Acad. Sci. U. S. A. 2015, 112, E25852594.

(33) Beglov, D.; Hall, D. R.; Wakefield, A. E.; Luo, L.; Allen, K. N.; Kozakov, D.; Whitty, A.; Vajda, S. Exploring the structural origins of cryptic sites on proteins. Proc. Natl. Acad. Sci. U. S. A. 2018, 115, E3416-E3425.

(34) Ghanakota, P.; Carlson, H. A. Driving structure-based drug discovery through cosolvent molecular dynamics. J. Med. Chem. 2016, 59, 10383-10399.

(35) Ung, P. M.; Ghanakota, P.; Graham, S. E.; Lexa, K. W.; Carlson, H. A. Identifying binding hot spots on protein surfaces by mixedsolvent molecular dynamics: HIV-1 protease as a test case. Biopolymers 2016, 105, 21-34.

(36) Kimura, S. R.; Hu, H. P.; Ruvinsky, A. M.; Sherman, W.; Favia, A. D. Deciphering cryptic binding sites on proteins by mixed-solvent molecular dynamics. J. Chem. Inf. Model. 2017, 57, 1388-1401.

(37) Arcon, J. P.; Defelipe, L. A.; Modenutti, C. P.; Lopez, E. D.; Alvarez-Garcia, D.; Barril, X.; Turjanski, A. G.; Marti, M. A. Molecular dynamics in mixed solvents reveals protein-ligand interactions, improves docking, and allows accurate binding free energy predictions. J. Chem. Inf. Model. 2017, 57, 846-863.

(38) Ghanakota, P.; van Vlijmen, H.; Sherman, W.; Beuming, T. Large-scale validation of mixed-solvent simulations to assess hotspots at protein-protein interaction interfaces. J. Chem. Inf. Model. 2018, 58, 784-793.

(39) Nguyen, H. C.; Yang, H.; Fribourgh, J. L.; Wolfe, L. S.; Xiong, Y. Insights into Cullin-RING E3 ubiquitin ligase recruitment: structure of the VHL-EloBC-Cul2 complex. Structure 2015, 23, 441-449.

(40) Silvestre, H. L.; Blundell, T. L.; Abell, C.; Ciulli, A. Integrated biophysical approach to fragment screening and validation for fragment-based lead discovery. Proc. Natl. Acad. Sci. U. S. A. 2013, 110, 12984-12989.

(41) Lepre, C. A.; Moore, J. M.; Peng, J. W. Theory and applications of NMR-based screening in pharmaceutical research. Chem. Rev. 2004, 104, 3641-3676.

(42) Cardote, T. A. F.; Ciulli, A. Structure-guided design of peptides as tools to probe the protein-protein interaction between Cullin-2 and elongin BC substrate adaptor in Cullin RING E3 ubiquitin ligases. ChemMedChem 2017, 12, 1491-1496.

(43) Dias, D. M.; Van Molle, I.; Baud, M. G.; Galdeano, C.; Geraldes, C. F.; Ciulli, A. Is NMR fragment screening fine-tuned to assess druggability of protein-protein interactions? ACS Med. Chem. Lett. 2014, 5, 23-28.

(44) Pastore, Y. D.; Jelinek, J.; Ang, S.; Guan, Y.; Liu, E.; Jedlickova, K.; Krishnamurti, L.; Prchal, J. T. Mutations in the VHL gene in sporadic apparently congenital polycythemia. Blood 2003, 101, 15911595.

(45) Russell, R. C.; Sufan, R. I.; Zhou, B.; Heir, P.; Bunda, S.; Sybingco, S. S.; Greer, S. N.; Roche, O.; Heathcote, S. A.; Chow, V. W.; Boba, L. M.; Richmond, T. D.; Hickey, M. M.; Barber, D. L.; Cheresh, D. A.; Simon, M. C.; Irwin, M. S.; Kim, W. Y.; Ohh, M. Loss of JAK2 regulation via a heterodimeric VHL-SOCS1 E3 ubiquitin ligase underlies Chuvash polycythemia. Nat. Med. 2011, 17, 845-853.

(46) Liu, J.; Nussinov, R. The mechanism of ubiquitination in the cullin-RING E3 ligase machinery: conformational control of substrate orientation. PLoS Comput. Biol. 2009, 5, e1000527.

(47) Woo, J. S.; Imm, J. H.; Min, C. K.; Kim, K. J.; Cha, S. S.; Oh, B. $\mathrm{H}$. Structural and functional insights into the B30.2/SPRY domain. EMBO J. 2006, 25, 1353-1363.
(48) Cardote, T. A. F.; Gadd, M. S.; Ciulli, A. Crystal structure of the Cul2-Rbx1-EloBC-VHL ubiquitin ligase complex. Structure 2017, 25, 901-911 e903.

(49) Lin, H. C.; Yeh, C. W.; Chen, Y. F.; Lee, T. T.; Hsieh, P. Y.; Rusnac, D. V.; Lin, S. Y.; Elledge, S. J.; Zheng, N.; Yen, H. S. Cterminal end-directed protein elimination by CRL2 ubiquitin ligases. Mol. Cell 2018, 70, 602-613 e603.

(50) Testa, A.; Lucas, X.; Castro, G.; Chan, K. H.; Wright, J.; Runcie, A.; Gadd, M.; Harrison, W. T. A.; Ko, E. J.; Fletcher, D.; Ciulli, A. 3-Fluoro-4-hydroxyprolines: synthesis, conformational analysis and stereoselective recognition by the VHL E3 ubiquitin ligase for targeted protein degradation. J. Am. Chem. Soc. 2018, 140, 9299. 